\title{
Dynamic characteristics of frequency-locked autodynes
}

\author{
Vladyslav Ya. Noskov", Kirill A. Ignatkov, and Kirill D. Shaidurov \\ UrFU, the Department R\&T IRIT-RTF, 620002, Yekaterinburg, Mira St, 19, Russia
}

\begin{abstract}
The results of research into the dynamic characteristics of microwave oscillators under the influence of both their own reflected radiation and external synchronising effect are presented. The basic relations for the analysis of signals during fast movement of the target are obtained, when the signal period is comparable to the autodyne response time constants. The results of numerical modelling of the characteristics are confirmed by the experimental data obtained on the example of an oscillator based on the Gunn diode of the 8-mm range.
\end{abstract}

\section{Introduction}

For the successful use of autodyne (AD) in various applications, knowledge about their dynamic characteristics is required, which should be taken into account in registering the parameters of various targets movement [1-6]. The dynamic properties of AD are determined by the type and width of their amplitude-frequency characteristic (AFC). To date, AFCs have been extensively studied in conventional AD [7-10]. It has been established that their AFC width is largely dependent on the time constant of the autodyne response $\tau_{\mathrm{a}}$ to a change in the oscillation amplitude which introduces a limitation on the maximum target velocity recorded by AD. However, in the synchronised AD (SAD), which is of practical interest, the results of studies of dynamic characteristics in the published literature [5] are unknown. In this regard, the aim of this work is to study the AFC transmission coefficients of the autodyne response by changing the amplitude and phase of the SAD oscillations depending on the speed of the location object by taking into account the internal parameters of the microwave oscillator and its synchronisation conditions.

\section{Mathematical Model for the Analysis and Calculation of the Dynamic Characteristics of Synchronised Autodyne}

In our previous work, Features of Autodyne Signals of Synchronised Microwave Oscillators, we developed a mathematical model of SAD with a single-circuit oscillatory system. In this case, the oscillator AD is under the influence of its own radiation reflected from the target and radiation coming from a third-party synchronising oscillator. A system of differential equations is obtained for relative changes in the amplitude $a$ detuning the

* Corresponding author: noskov@oko-ek.ru 
frequencies of external radiation $\omega_{\mathrm{ex}}$ and a stand-alone oscillator $\omega_{0}$, and also for absolute changes in the SAD phase $\tilde{\varphi}$ as follows:

$$
\begin{aligned}
& \frac{d a}{d t}+\frac{1}{\tau_{\mathrm{a}}} a+\frac{\operatorname{tg} \varphi_{0}}{\tau_{\varphi}} \tilde{\varphi}+\rho\left(\omega_{\mathrm{ex}}-\omega_{0}\right)=\Gamma \frac{K_{\mathrm{a}}}{\tau_{\mathrm{a}}} \cos \delta(t, \tau), \\
& \frac{d \tilde{\varphi}}{d t}+\frac{\gamma}{\tau_{\mathrm{a}}} a+\frac{1}{\tau_{\varphi}} \tilde{\varphi}+\left(\omega_{\mathrm{ex}}-\omega_{0}\right)=-\Gamma \frac{K_{\mathrm{a}}}{\tau_{\mathrm{a}}} \sin \delta(t, \tau),
\end{aligned}
$$

where $K_{\mathrm{a}}=\eta / \alpha$ is autodyne growth rate; $\tau_{\mathrm{a}}, \tau_{\varphi}$ is the time constants of changes in the amplitude and phase, respectively:

$$
\tau_{\mathrm{a}}=Q_{\mathrm{L}} / \alpha \omega_{0}, \tau_{\varphi}=Q_{\mathrm{ex}} / k_{\mathrm{ex}} \omega_{0} \cos \varphi_{0}
$$

$\gamma=\beta / \alpha, \rho=\varepsilon / Q_{\mathrm{L}}$ is $\mathrm{SAD}$ an isochronous and non-isodromic coefficients, respectively; $\alpha, \varepsilon, \beta$ are parameters characterising the strength of the limit cycle, non-isodromy and non-isochronism of the oscillator, respectively; $\eta=Q_{\mathrm{L}} / Q_{\mathrm{ex}}, Q_{\mathrm{L}}, Q_{\mathrm{ex}}$ is efficiency loaded and external Q of OS; $\Gamma, k_{\mathrm{ex}}$ are coefficients characterising the relative levels in the case of load on the oscillator received from the oscillation target and the external synchronising effect, respectively; $\varphi_{0}$ is a phase of steady-state SAD oscillations in the absence of radiation from the target when $\Gamma=0$ :

$$
\varphi_{0}=-\left(\theta_{0}+\arcsin \chi_{\mathrm{n}}\right)
$$

$\theta_{0}=\operatorname{arctg} \gamma$ is phase characteristic displacement angle; $\chi_{\mathrm{n}}=\left(\omega_{\mathrm{ex}}-\omega_{0}\right) / \Delta_{m}$ is specified frequency detuning $\omega_{\mathrm{ex}}$ and $\omega_{0} ; \Delta_{m}$ is half-width of the oscillator synchronisation band:

$$
\Delta_{m}=k_{\mathrm{ex}} \frac{\omega_{0}}{Q_{\mathrm{ex}}} \frac{\left(1+\gamma^{2}\right)^{1 / 2}}{(1-\gamma \rho)} .
$$

To determine the dynamic characteristics of SAD, we set in the system of equations (1), (2) the initial conditions for the interaction between the oscillator and microwave radiation reflected from the moving target, when $\tau \equiv \tau(t)$. Given the constancy of the speed of the phase incursion $\delta(t, \tau)$, we write as follows:

$$
\delta(t, \tau)=\omega_{\mathrm{ex}} \tau(t)=\Omega_{\mathrm{D}} t+\delta_{0}
$$

where $\delta_{0}=\omega_{\mathrm{ex}} \tau_{0}$ is the initial phase of the Doppler signal, which in the future we assume to be equal to zero. After transforming the system (1), (2) taking into account (6) and excluding the constant component from the analysis, we obtain two independent secondorder equations for relative changes in amplitude $a$ and absolute changes in phase $\tilde{\varphi}$ as follows:

$$
\frac{d^{2} a}{d t^{2}}+\frac{\tau_{\mathrm{a}}+\tau_{\varphi}}{\tau_{\mathrm{a}} \tau_{\varphi}} \frac{d a}{d t}+\frac{1-\gamma \operatorname{tg} \varphi_{0}}{\tau_{\mathrm{a}} \tau_{\varphi}} a=\Gamma \frac{K_{\mathrm{a}}}{\tau_{\mathrm{a}}} \frac{1}{\tau_{\varphi}}\left[\cos \Omega_{\mathrm{D}} t+\left(\operatorname{tg} \varphi_{0}-k_{\Omega} \Omega\right) \sin \Omega_{\mathrm{D}} t\right],
$$




$$
\frac{d^{2} \tilde{\varphi}}{d t^{2}}+\frac{\tau_{\mathrm{a}}+\tau_{\varphi}}{\tau_{\mathrm{a}} \tau_{\varphi}} \frac{d \tilde{\varphi}}{d t}+\frac{1-\gamma \operatorname{tg} \varphi_{0}}{\tau_{\mathrm{a}} \tau_{\varphi}} \tilde{\varphi}=-\Gamma \frac{K_{\mathrm{a}}}{\tau_{\mathrm{a}}} \frac{1}{\tau_{\mathrm{a}}}\left[\sin \Omega_{\mathrm{D}} t+(\gamma+\Omega) \cos \Omega_{\mathrm{D}} t\right]
$$

where $k_{\Omega}=\Omega_{\varphi} / \Omega=1 / U_{\mathrm{ex}} \cos \varphi_{0}$ is the coefficient characterising the ratio of the specified frequencies $\Omega=\tau_{\mathrm{a}} \Omega_{\mathrm{D}}$ и $\Omega_{\varphi}=\tau_{\varphi} \Omega_{\mathrm{D}} ; U_{\text {ex }}=k_{\mathrm{ex}} K_{\mathrm{a}}$ is a relative level of the external influence by taking into account its autodyne amplification.

The right-hand sides of equations (7), (8) are the trigonometric functions of time. Therefore, we seek general solutions of these equations in the form of functions, $a(t)=Z_{\mathrm{s}} \sin \Omega_{\mathrm{D}} t+Z_{\mathrm{c}} \cos \Omega_{\mathrm{D}} t$ and $\tilde{\varphi}(t)=W_{\mathrm{s}} \sin \Omega_{\mathrm{D}} t+W_{\mathrm{c}} \cos \Omega_{\mathrm{D}} t$, where $Z_{\mathrm{s}}, Z_{\mathrm{c}}, W_{\mathrm{s}}, W_{\mathrm{c}}$ are the sought factors for the sine (index ' $\mathrm{s}$ ') and cosine (index 'c'). After a series of elementary transformations of the obtained solutions, we write the final expressions for the steady-state values of the dynamic changes in phase $\tilde{\varphi}(t)$ and amplitude $a(t)$ SAD:

$$
\begin{gathered}
\tilde{\varphi}(t)=-\left(\Gamma / k_{\mathrm{ex}} \cos \varphi_{0}\right) H_{\varphi} \Phi_{\mathrm{d}}(\Omega) \sin \left[\Omega_{\mathrm{D}} t+\theta_{\mathrm{d}}(\Omega)\right], \\
a(t)=\Gamma K_{\mathrm{a}} H_{\mathrm{a}} K_{д}(\Omega) \cos \left[\Omega_{\text {д }} t+\psi_{\text {д }}(\Omega)\right],
\end{gathered}
$$

where $\Phi_{\mathrm{d}}(\Omega), K_{\mathrm{d}}(\Omega)$ are 'dynamic' AFC SAD by changing the oscillations phase and amplitude, respectively:

$$
\begin{aligned}
& \Phi_{\mathrm{d}}(\Omega)=\sqrt{w_{\mathrm{s}}^{2}(\Omega)+w_{\mathrm{c}}^{2}(\Omega)}, \\
& K_{\mathrm{d}}(\Omega)=\sqrt{z_{\mathrm{s}}^{2}(\Omega)+z_{\mathrm{c}}^{2}(\Omega)},
\end{aligned}
$$

$\theta_{d}(\Omega), \psi_{d}(\Omega)$ are the angles of the 'dynamic' shift of phase changes and relative changes in the amplitude of the SAD oscillations, respectively:

$$
\begin{aligned}
& \theta_{\mathrm{d}}(\Omega)=\operatorname{arctg}\left[w_{\mathrm{c}}(\Omega) / w_{\mathrm{s}}(\Omega)\right], \\
& \psi_{\mathrm{d}}(\Omega)=\operatorname{arctg}\left[z_{\mathrm{s}}(\Omega) / z_{\mathrm{c}}(\Omega)\right],
\end{aligned}
$$

$z_{\mathrm{s}}(\Omega), z_{\mathrm{c}}(\Omega), w_{\mathrm{s}}(\Omega), w_{\mathrm{c}}(\Omega)$ are the quadrature factors normalised relative to the values $H_{\mathrm{a}}$ and $H_{\varphi}$ :

$$
\begin{aligned}
& z_{\mathrm{s}}(\Omega)=\frac{Z_{\mathrm{s}}}{H_{\mathrm{a}}}=\frac{\left(\operatorname{tg} \varphi_{0}-k_{\Omega} \Omega\right)\left(1-\gamma \operatorname{tg} \varphi_{0}-k_{\Omega} \Omega^{2}\right)+\left(1+k_{\Omega}\right) \Omega}{H_{\mathrm{a}}\left[\left(1-\gamma \operatorname{tg} \varphi_{0}-k_{\Omega} \Omega^{2}\right)^{2}+\left(1+k_{\Omega}\right)^{2} \Omega^{2}\right]}, \\
& z_{\mathrm{c}}(\Omega)=\frac{Z_{\mathrm{c}}}{H_{\mathrm{a}}}=\frac{\left(1-\gamma \operatorname{tg} \varphi_{0}-k_{\Omega} \Omega^{2}\right)-\left(\operatorname{tg} \varphi_{0}-k_{\Omega} \Omega\right)\left(1+k_{\Omega}\right) \Omega}{H_{\mathrm{a}}\left[\left(1-\gamma \operatorname{tg} \varphi_{0}-k_{\Omega} \Omega^{2}\right)^{2}+\left(1+k_{\Omega}\right)^{2} \Omega^{2}\right]}, \\
& w_{\mathrm{s}}(\Omega)=\frac{W_{\mathrm{s}}}{H_{\varphi}}=\frac{\left(1-\gamma \operatorname{tg} \varphi_{0}-k_{\Omega} \Omega^{2}\right)+(\gamma+\Omega)\left(1+k_{\Omega}\right) \Omega}{H_{\varphi}\left[\left(1-\gamma \operatorname{tg} \varphi_{0}-k_{\Omega} \Omega^{2}\right)^{2}+\left(1+k_{\Omega}\right)^{2} \Omega^{2}\right] \cos \varphi_{0}}, \\
& w_{\mathrm{c}}(\Omega)=\frac{W_{\mathrm{c}}}{H_{\varphi}}=\frac{(\gamma+\Omega)\left(1-\gamma \operatorname{tg} \varphi_{0}-k_{\Omega} \Omega^{2}\right)-\left(1+k_{\Omega}\right) \Omega}{H_{\varphi}\left[\left(1-\gamma \operatorname{tg} \varphi_{0}-k_{\Omega} \Omega^{2}\right)^{2}+\left(1+k_{\Omega}\right)^{2} \Omega^{2}\right] \cos \varphi_{0}},
\end{aligned}
$$


The analysis of expressions (9) - (18), due to their complexity, is performed numerically by introducing parameter variations. At the same time, we will take into account that in SAD, the values $\tau_{\mathrm{a}}$ and, respectively, $\Omega=\tau_{\mathrm{a}} \Omega_{\mathrm{D}}$ are independent of the synchronisation mode. However, the time constant $\tau_{\varphi}$ depending on the synchronisation conditions can vary widely. As can be seen from (3), it has the lowest value $\tau_{\varphi}^{\min }=Q_{\mathrm{ex}} / k_{\mathrm{ex}} \omega_{0}$ in the centre of the synchronisation band and increases sharply at its edges. In addition, the value $\tau_{\varphi}$ depends on the relative level $k_{\text {ex }}$ of the external synchronising effects.

Figures 1 and 2 show the results of calculations in the form of AFC diagrams of the transfer coefficients of the autodyne changes in the phase $\Phi_{\mathrm{d}}(\Omega)$ and amplitude $K_{\mathrm{d}}(\Omega)$ of the SAD oscillations according to (11) and (12) for various values of the parameters $k_{\Omega}$, $U_{\text {ex }}, \chi_{\mathrm{n}}$ and $\gamma$.

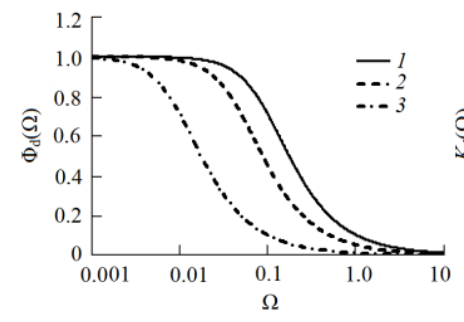

(a)

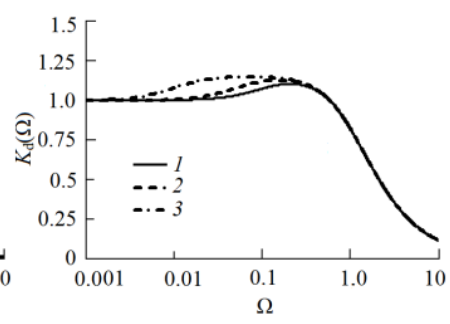

(b)

Fig. 1. Diagrams AFC $\Phi_{\mathrm{d}}(\Omega)(a)$ and $K_{\mathrm{d}}(\Omega)(b)$, calculated for $\gamma=0, \chi_{\mathrm{n}}=0$ and when the level of external influence is varied $U_{\mathrm{ex}}: U_{\mathrm{ex}}=0.1$ (curve 1), $U_{\mathrm{ex}}=0.05$ (curve 2), $U_{\mathrm{ex}}=0.01$ (curve 3).

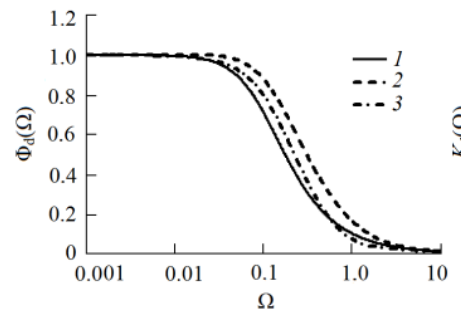

(a)

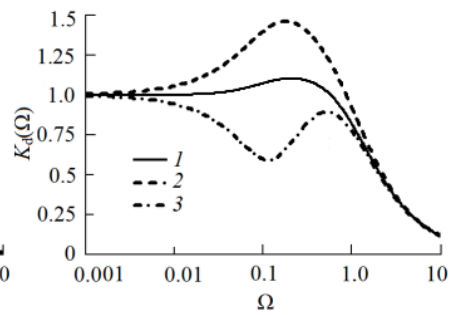

(b)

Fig. 2. Diagrams AFC $\Phi_{\mathrm{d}}(\Omega)(a)$ and $K_{\mathrm{d}}(\Omega)(b)$, calculated for $U_{\mathrm{ex}}=0.1, \chi_{\mathrm{n}}=0$ and coefficient variations $\gamma: \gamma=0$ (curve 1 ), $\gamma=1$ (curve 2), $\gamma=-1$ (curve 3).

From Figures $1 \mathrm{a}$ and $2 \mathrm{a}$, as well as the calculation results for other values of the parameters $k_{\Omega}, U_{\text {ex }}, \chi_{\mathrm{n}}$ and $\gamma$ it follows that the AFC of the autodyne change transmission coefficients in the $\Phi_{\mathrm{d}}(\Omega)$ phase of SAD are the monotonic functions of frequency. If the frequency $\Omega$ of the autodyne signal of the boundary value $\Omega_{\lim }^{\varphi}$ is exceeded, the transfer coefficient $\Phi_{\mathrm{d}}(\Omega)$ decreases sharply. This decrease in AFC is due to the inertia of the oscillator synchronisation process, in which the phase of oscillations of the synchronised oscillator does not have time to respond to rapid changes in the $\delta(t, \tau)$ phase incursion. In this case, the boundary frequency of the transfer coefficient $\Phi_{d}(\Omega)$ in terms of level $1 / 2^{1 / 2}$ is determined by the time constant $\tau_{\varphi}$, as in a regular inertial link of the first order: 


$$
\Omega_{\lim }^{\varphi}=1 / \tau_{\varphi}=k_{\mathrm{ex}}\left(\omega_{0} / Q_{\mathrm{ex}}\right) \cos \varphi_{0},
$$

From the analysis of diagrams of Figures $1, b$ and 2, $b$ it follows that the AFC of the transfer coefficient $K_{\mathrm{d}}(\Omega)$ of the autodyne changes in the amplitude of the SAD oscillations are not monotonic functions of the frequency $\Omega$ of the autodyne response. The form of those functions is largely determined by the synchronisation conditions (the values of the $U_{\text {ех }}$ and $\chi_{\text {н }}$ parameters), the ratio $k_{\Omega}$ of the time constants $\tau_{\mathrm{a}}$ and $\tau_{\varphi}$, and also by the value and sign of the non-isochronism coefficient $\gamma$.

The reason for the non-uniformity of the AFC transfer coefficient $K_{\mathrm{d}}(\Omega)$ is associated with the internal 'interference' of the components of the autodyne response to a change in the amplitude of the oscillations. The first component is associated with the conversion of changes in the phase $\tilde{\varphi}(t)$ of the microwave oscillator into changes in the amplitude of its oscillations (see the third term in the left-hand side of (1)). The second component is due to changes in the phase incursion $\delta(t, \tau)$ of the reflected microwave radiation (see the righthand side of (1)). The dynamic $\theta_{\mathrm{d}}(\Omega)$ phase displacements of the autodyne response depending on the frequency $\Omega$, as shown by calculations according to (13), cause changes in the phase relations between these terms. Therefore, the resulting response to a change in the amplitude $a(t)$ of oscillations increases at those frequencies at which the in-phase components and attenuates at frequencies with antiphase terms.

\section{Experimental Results}

The 8-mm 'Tigel-08M' hybrid-integrated oscillator module based on the Gunn planar diode was used as a target of research. Its supply voltage is $4 \mathrm{~V}$, with a consumption current of about $0.2 \mathrm{~A}$. The module generation frequency is $37.5 \mathrm{GHz}$, the output power is $15 \mathrm{~mW}$. The power of the synchronising signal was $1 \mathrm{~mW}$. To register a signal by changing the oscillation amplitude, a detector diode with a Schottky barrier is built into the topology of the module [11].

For the experimental research into AFC SAD in a wide frequency range, the indirect method known as the 'modulation characteristics' method was used [12,13]. It is indirect because direct measurements of the characteristics using types of Doppler signal simulators known to us are impossible because of their limited speed of response. For this, the simulator was replaced with a reflection diode modulator [14]. The operating point on the modulator diode characteristics was selected in the middle of the linear portion of its forward bias. A modulating signal of the level of $2 \mathrm{dBm}$ was applied to the diode through an isolation capacitor from the output of the 'Obzor-103' complex transfer coefficient meter. In this case, an autodyne signal from the output of the detector diode of the 'Tigel$08 \mathrm{M}$ ' oscillator module was applied to the input of the meter. The length of the waveguide path from $\mathrm{AD}$ to the diode modulator was $0.22 \mathrm{~m}$, the attenuator attenuation in this path was set to about $15 \mathrm{~dB}$, and the analysed frequency range $\Omega / 2 \pi$ was set from 0.3 to 300 $\mathrm{MHz}$.

For the case when the frequency $\omega_{\text {вн }}$ of the 'external' synchronising signal corresponds to the middle of the synchronisation band $\omega_{0}$, AFC is obtained in the form of curve 2. For cases, where there are initial frequency detuning $\omega_{\text {вн }}$ up and down relative to frequency $\omega_{0}$ by value $\chi_{\mathrm{H}}=0.8$, obtained are AFCs represented by curves 3 and 4 , respectively. 


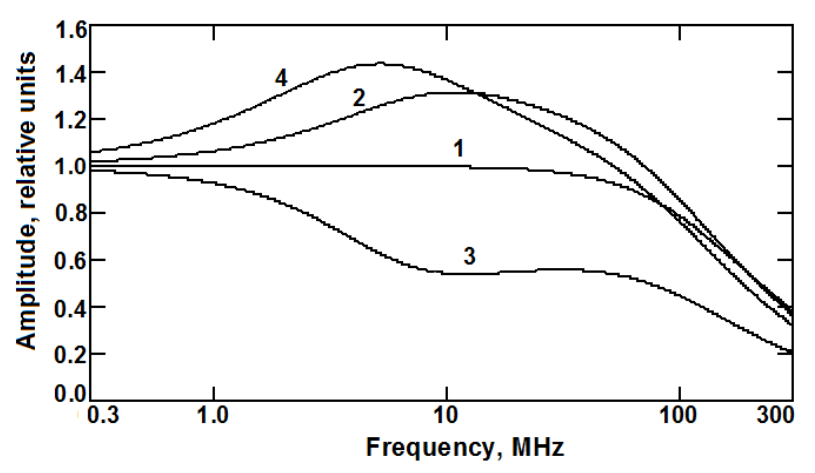

Fig. 3. AFC of the autodyne response transfer coefficients for a change in the amplitude of the oscillations obtained experimentally for $\mathrm{AD}$ on the Gunn diode if there is no synchronisation (curve 1 ) and if it is available for cases, when $\chi_{\mathrm{H}}=0$ (curve 2), $\chi_{\mathrm{H}}=-0.8$ (curve 3) and $\chi_{\mathrm{H}}=0.8$ (curve 4).

AFC of the oscillator module without synchronisation (see curve 1 ) is uniform in a wide frequency range. The boundary frequency of this characteristic in level $1 / 2^{1 / 2}$ is about 120 MHz. In this case, the value of the time constant $\tau_{\mathrm{a}}$ of the amplitude changes is obtained $1.3 \times 10^{-9} \mathrm{~s}$. This value $\tau_{\mathrm{a}}$ is consistent with the data presented in $[12,13]$. In the case that $\mathrm{SAD}$ of the AFC module, as can be seen from curves 2 - 4 in Fig. 3 may have significant non-uniformity in the range of units and tens of $\mathrm{MHz}$. The boundary frequency of the frequency response, under number ' 3 ', was obtained on the order of $1.2 \ldots 3 \mathrm{MHz}$. It should be noted that this frequency range with uniform $\mathrm{AFC}$ is quite enough to record the movements of location objects to the speeds of $4.8 \ldots 12 \mathrm{~km} / \mathrm{s}$.

Thus, the experimental research results obtained in this work confirmed the adequacy of the developed mathematical model of SAD in relation to the analysis of autodyne parameters and characteristics at high target speeds.

\section{Conclusion}

A mathematical model of AD stabilised in frequency by a signal from an additional generator is developed. The basic relationships are obtained for calculating its signal characteristics at high target speeds, when the autodyne signal period is commensurate with the autodyne response time constants. It is shown that SAD can be used to analyse the motion parameters of high-speed targets. It was found that the inertia of the phase synchronisation process of the oscillator causes uneven formation of the AFC of the synchronised AD transmission coefficient by a change in the amplitude of oscillations in the high-frequency region. However, this non-uniformity with the correct choice of synchronisation parameters is not an obstacle to registering signals in the entire range of target speeds in practice.

From the research results it follows that it is quite promising to develop integrated autodyne modules with frequency synchronisation from an additional low-power oscillator.

\section{References}

1. D.A. Usanov, Al.V. Skripal, An.V. Skripal, A.E. Postelga. Instrum. \& Experimental Techn. 47, 5. (2004)

2. S.A. Alidoost, R. Sadeghzade, R. Fatemi. 11-th Intern. Radar Symp. (IRS-2010). Vilnius, Lithuania. 2, 406-409 (2010) 
3. D.A. Usanov, A.E. Postelga. Biomedical Engineering. 45, 1. (2011)

4. G.P. Ermak, I.V. Popov, A.S. Vasiliev, A.V. Varavin, V.Ya. Noskov, K.A. Ignatkov. Telecommunication \& Radio Engineering. 71, 6. (2012)

5. I.V. Komarov, S.M. Smolskiy. Fundamentals of Short-Range FM Radar. Artech House, Norwood, MA, USA (2003)

6. V.Ya. Noskov, K.A. Ignatkov, A.P. Chupahin, et al. Radioengineering. 26, 4. (2017)

7. S.D. Votoropin, V.Ya. Noskov. Russian Physics Journal. 43, 7. (2000)

8. V.Ya. Noskov, K.A. Ignatkov. 22-th International Crimean Conference "Microwave \& Telecommunication Technology". Sevastopol. 2. P. 883. (2012)

9. V.Ya. Noskov, K.A. Ignatkov. 22-th International Crimean Conference "Microwave \& Telecommunication Technology". Sevastopol. 2. P. 885. (2012)

10. V.Ya. Noskov, K.A. Ignatkov. Russian Physics Journal. 56, 4. (2013)

11. V.Ya. Noskov, K.A. Ignatkov. Journal of Communications Technology and Electronics. 61, 9. (2016)

12. V.Ya. Noskov, K.A. Ignatkov. Telecommunication \& Radio Engineering. 72, 10. (2013)

13. V.Ya. Noskov, K.A. Ignatkov, S.M. Smolskiy. Telecommunication Sciences. 3, 2. (2012)

14. V.Ya. Noskov, K.A. Ignatkov. 22-th International Crimean Conference "Microwave \& Telecommunication Technology". Sevastopol. 2. P. 887. (2012) 